\title{
The configuration of simple short-duration solar microwave bursts
}

\author{
M. R. Kundu ${ }^{1}$, A. Nindos ${ }^{2}$, and V. V. Grechnev ${ }^{3}$ \\ 1 Astronomy Department, University of Maryland, College Park, MD 20742, USA \\ e-mail: kundu@astro.umd.edu \\ 2 Section of Astrogeophysics, Physics Department, University of Ioannina, Ioannina 45110, Greece \\ e-mail: anindos@cc.uoi.gr \\ 3 Institute of Solar-Terrestrial Physics, Lermontov St. 126, Irkutsk 664033, Russia \\ e-mail: grechnev@iszf.irk.ru
}

Received 7 October 2003 / Accepted 2 March 2004

\begin{abstract}
Using data from the Nobeyama Radioheliograph (NoRH) we study the source configuration of four simple short-duration 17 and $34 \mathrm{GHz}$ bursts which have also been observed partially by the Yohkoh Soft X-ray Telescope (SXT). Two events are consistent with a single flaring loop configuration. In one of them the flaring loop is resolved in the SXT images. We derive a self-consistent model for this event by comparing the radio observations with gyrosynchrotron model loop calculations. Our best-fit model is able to reproduce both the observed flaring loop shape as well as the fluxes and structures of the radio emission at the peak of the event. The flaring loop is relatively small having a footpoint separation of $16^{\prime \prime}$ and maximum height of 7.7". The variation of the magnetic field along the loop is small ( $800 \mathrm{G}$ at the footpoints and $665 \mathrm{G}$ at loop top) and the loop is filled with electrons with energies up to $10 \mathrm{MeV}$. The other two bursts show two radio sources; one source being cospatial with a compact bright soft X-ray loop associated with a patch of parasitic magnetic polarity whose photospheric magnetic flux increases before the flares while the other source is not prominent at any other wavelength range. The two sources are connected with diffuse loop-like soft X-ray emission. We infer that these bursts originate from the interaction of two sets of loops with different sizes. Therefore the simple short duration microwave bursts we studied do not always appear in the same configuration. Contrary to previous results not all of them appear as single-loop events. It is possible that some events are caused by two interacting loops.
\end{abstract}

Key words. Sun: corona - Sun: radio radiation - Sun: flares - Sun: X-rays, gamma rays

\section{Introduction}

Microwave emission from solar flares comes from electrons accelerated during the flare. Through the gyrosynchrotron mechanism, mildly relativistic electrons (energies of tens to several hundreds of $\mathrm{keV}$ ) emit at microwaves as they spiral along magnetic field lines in the corona (e.g. Dulk 1985; Alissandrakis 1986; Bastian et al. 1998). The gyrosynchrotron emission mechanism depends intrinsically on the coronal magnetic field strength and orientation; therefore suitable observations of microwave bursts may be used to constrain the coronal magnetic field configuration in the flaring source.

Simultaneous microwave, soft X-ray and EUV flare observations show that the magnetic configuration of the flaring region can be consistent with a single loop (e.g. White et al. 2002), interacting loops of different sizes (e.g. Hanaoka 1997) or more complex loop systems (e.g. Kundu et al. 1982). At microwaves, for a given frequency the morphology of the radio

Send offprint requests to: A. Nindos, e-mail: anindos@cc.uoi.gr source depends on the geometry of the flaring region and its location on the disk, as well as the properties of the nonthermal electrons. At high frequencies the emission is presumably optically thin and loop footpoints are seen (e.g., Shevgaonkar \& Kundu 1985; Bastian \& Kiplinger 1991; Alissandrakis et al. 1993; Nishio et al. 1997) while at low frequencies a portion of the loop top is optically thick and dominates the emission (e.g. Holman et al. 1982; Shevgaonkar \& Kundu 1985; Bastian \& Kiplinger 1991).

The above picture comes primarily from events which show a relatively impulsive rise (i.e. the peak radio emission is reached within a few seconds to $2-3$ min from the onset of flare) followed by a much longer decay phase. Using the Nobeyama Radioheliograph (NoRH) at 17 and $34 \mathrm{GHz}, \mathrm{Kundu}$ et al. (2001c) studied the spatial structure of five short duration simple spiky bursts with fast $2-6 \mathrm{~s}$ rise time to peak, followed by a rapid exponential decay. Such time profiles are similar to those found to be common at $86 \mathrm{GHz}$ bursts observed with the Berkeley-Illinois-Maryland Association (BIMA) interferometer (e.g., see White 1994). The source sizes of four of the bursts 
Table 1. Simple short-duration bursts.

\begin{tabular}{|c|c|c|c|c|c|c|c|c|}
\hline Date & $\begin{array}{l}\text { Peak time } \\
\text { (UT) }\end{array}$ & $\begin{array}{c}\text { Duration }^{a} \\
\quad(\mathrm{sec})\end{array}$ & Location & $\begin{array}{l}\text { GOES } \\
\text { Class. }\end{array}$ & $\begin{array}{c}\text { Flux } \\
17 \mathrm{GHz} \\
\text { (SFU) }\end{array}$ & $\begin{array}{c}T_{b}^{\max } \\
17 \mathrm{GHz} \\
\left(\times 10^{6} \mathrm{~K}\right)\end{array}$ & $\begin{array}{c}\text { Flux } \\
34 \mathrm{GHz} \\
\text { (SFU) }\end{array}$ & $\begin{array}{c}T_{b}^{\max } \\
34 \mathrm{GHz} \\
\left(\times 10^{6} \mathrm{~K}\right)\end{array}$ \\
\hline 1999 July 30 & $22: 58: 09$ & 4 & N24E38 & C3.7 & 18 & 6.1 & 9 & 2.3 \\
\hline 2000 July 13 & $05: 24: 48$ & 7 & N18W72 & C5.3 & 3.1 & 0.4 & 1.4 & 0.1 \\
\hline 2000 July 13 & $05: 40: 22$ & 9 & N18W72 & C3.6 & 6.3 & 0.7 & 3.2 & 0.2 \\
\hline 2000 July 13 & $06: 11: 21$ & 18 & N18W71 & C6.0 & 46 & 7.6 & 23 & 2.5 \\
\hline
\end{tabular}

${ }^{a}$ FWHM of the time profile of the short duration burst's $17 \mathrm{GHz}$ flux.

studied were less than $5^{\prime \prime}$ and three of them showed closely spaced oppositely polarized components in the circular polarization $(V)$ maps. The total intensity $(I)$ in all five events peaked directly over photospheric magnetic neutral lines. Their spatial features and fast time profiles led Kundu et al. (2001c) to infer that these flares occur in compact single loops and therefore the acceleration of nonthermal electrons is confined to a compact localized region.

The study by Kundu et al. (2001c) used only radio and photospheric magnetic field data in order to infer the spatial properties of simple short-duration events. The goal of our paper is to derive a comprehensive view about the configuration and properties of such events by using NoRH microwave data and soft X-ray images obtained from the SXT. The two data sets complement each other: the microwave emission is more sensitive to the magnetic field and the properties of the energetic electrons while the SXT images reveal better the geometry of the flaring loops and give information about the thermal plasma. Using data from both instruments, in one event we are able to derive a self-consistent quantitative model about the magnetic field configuration of the flaring region and the properties of the nonthermal electrons.

\section{Observations and data analysis}

Inspection of the NoRH correlation plots (effectively time profiles of the small spatial scale flux at 17 and $34 \mathrm{GHz}$ ) shows that simple short-duration events are commonly detected and this approach was used to identify a suitable sample. However, for our study we require that SXT images are available at least for part of the events. This criterion rules out most of them because (1) the duration of the events is short, and (2) according to GOES soft X-ray data their soft X-ray emission is usually weak. The SXT observes in "flare mode" (i.e. high cadence and high spatial resolution) when it detects a bright feature anywhere on the solar disk, in which case its pointing shifts to this target. This is the reason why SXT rarely observes the soft X-ray counterparts of NoRH simple short-duration events. Therefore it is not surprising that only four such events met our criteria after we searched a database of Nobeyama and SXT observations over a period of one year.

The NoRH consists of 84 antennas in a T-shaped array (Nakajima et al. 1994). It observes the full solar disk in two circular polarizations at $17 \mathrm{GHz}$ and one linear polarization at $34 \mathrm{GHz}$. The NoRH data presented here were processed in the
NRAO sofware package AIPS. Since in our study it is crucial to exploit fully the spatial resolution available in the NoRH data, the final images were made using uniform weighting, cleaned, self-calibrated and then restored with beam sizes of $11^{\prime \prime}$ at $17 \mathrm{GHz}$ and $8^{\prime \prime}$ at $34 \mathrm{GHz}$. The time resolution of our maps was $1 \mathrm{~s}$.

Longitudinal photospheric magnetograms from the Michelson Doppler Imager (MDI) and EUV images from the Extreme Ultraviolet Incidence Telescope (EIT) on the "Solar and Heliospheric Observatory" spacecraft (SOHO) were used for context information. Unfortunately no data from the TRACE satellite were available for our events. The coregistration of the different data sets was done using the known pointing information of the instruments and as reference time we used the time of the NoRH peaks. The resulting overlays were checked by comparing the extended preflare thermal emission which appears in the soft X-ray, EUV and $17 \mathrm{GHz}$ images. The accuracy of the overlays of the radio images on the SXT/MDI/SXT images is not generally better than $5^{\prime \prime}$. The properties of the events we studied are summarized in Table 1.

\section{Results}

\subsection{Event of 1999 July 30}

This event has been presented by Kundu et al. (2001c). Here we also include soft X-rays images in our analysis and we present it in Fig. 1 (Kundu et al. 2001c studied only microwave data from this event). The 17 and $34 \mathrm{GHz} I$ maps peak between the two sources in the $V$ map over a photospheric magnetic field neutral line. From these features Kundu et al. (2001c) inferred that the radio emission comes from a compact bipolar loop. The SXT images strengthen this interpretation. They show (the first partial frame image, PFI, available appears in Fig. 1) that the radio event is associated with a bright compact loop which remains unresolved until the end of the flare.

From the SXT images we calculate the temperature $T_{\mathrm{e}}$ and emission measure $E M$ of the flaring loop at the decay phase of the radio event. In our analysis we have ignored all pixels with errors $\mathrm{d} \log E M>0.25$ and $\mathrm{d} \log T_{\mathrm{e}}>0.07$. The first set of $T_{\mathrm{e}}$ and $E M$ maps is obtained at 22:58:27 UT and shows that the average temperature of the compact loop is about $11.5 \mathrm{MK}$. From the derived emission measure and the size of the loop we obtain that the average plasma density of the loop is $N_{\mathrm{e}} \approx$ $10^{11} \mathrm{~cm}^{-3}$. Then both $T_{\mathrm{e}}$ and $N_{\mathrm{e}}$ gradually decrease. 


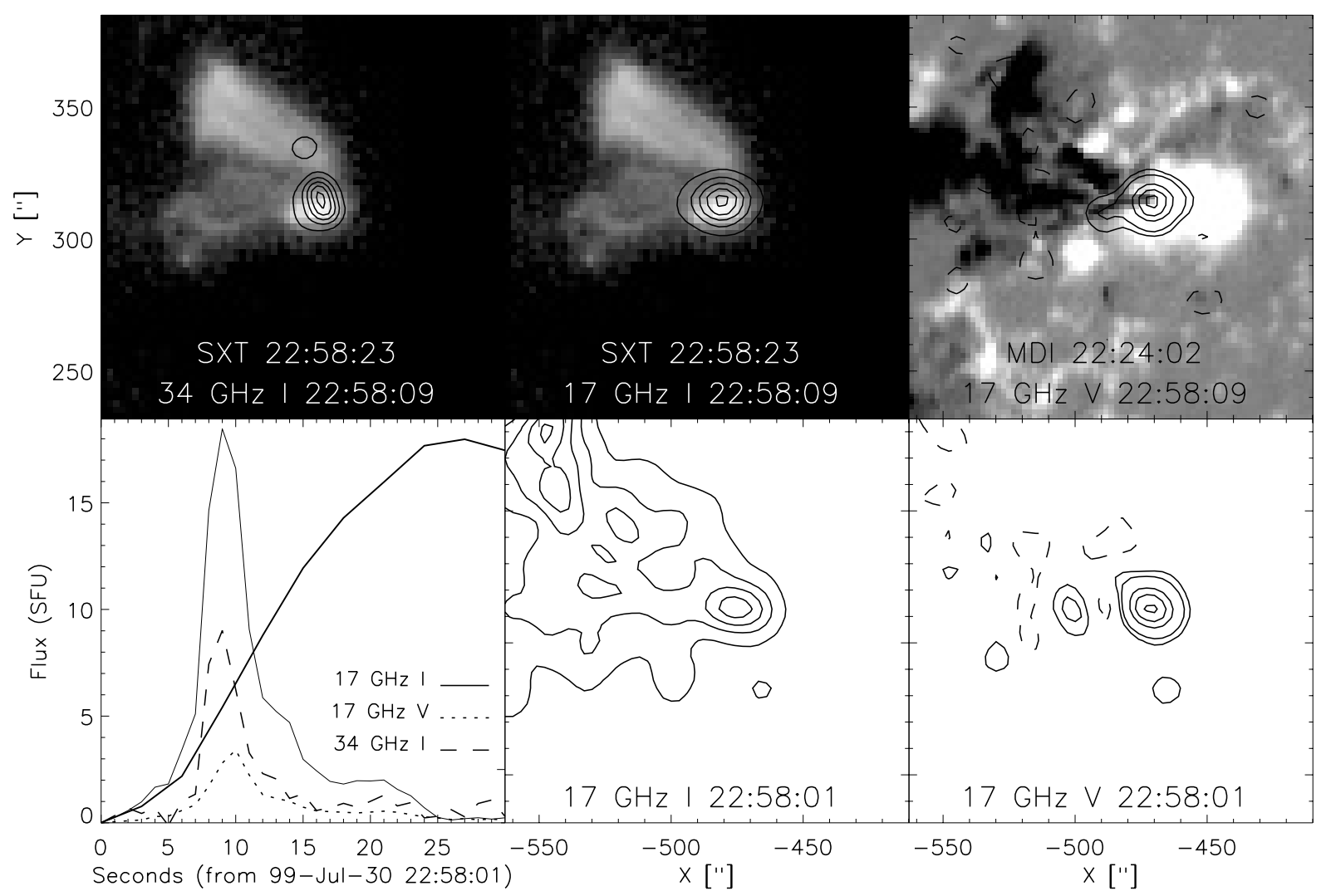

Fig. 1. The 1999 July 30 event. Top row from left to right: $34 \mathrm{GHz} I, 17 \mathrm{GHz} I$ and $V$ images at the peak of the radio burst overlaid on an SXT image and an MDI magnetogram. Contours are plotted at $\pm 15 \%, 35 \%, 55 \%, 75 \%$, and $95 \%$ of the respective peaks. In the $V$ map (maximum of $8 \times 10^{5} \mathrm{~K}$ ) solid lines indicate positive polarization and dashed lines indicate negative polarization. Bottom row left: time profiles of the radio flux at $17 \mathrm{GHz} I$, and $V$ and $34 \mathrm{GHz} I$ and time profile of the soft X-ray flux measured by the GOES satellite in the $1.5-10 \mathrm{keV}$ channel (thick solid line). Bottom middle and right: Preflare images of the $17 \mathrm{GHz} I$ and $V$ emission. Contours are plotted at $\pm 15 \%, 35 \%, 55 \%$, $75 \%$ of the maximum $I$ and $V$ maps $\left(4 \times 10^{5} \mathrm{~K}\right.$ and $2.3 \times 10^{5} \mathrm{~K}$, respectively). In all images North is up and West to the right. The axes labels denote seconds of arc from disk center. Here and in Figs. 2 and 5 the radio flare images are presented after the preflare radio emission has been subtracted out.

\subsection{Events of 2000 July $13,05: 25$ and $05: 40$ UT}

These two events occur at the same location in AR NOAA 9070. Images from the events are presented in Fig. 2. Their 17 and $34 \mathrm{GHz} I$ emissions consist of two components. The western components are essentially unpolarized and are associated with a small compact soft X-ray loop. This loop is associated with a patch of parasitic negative polarity (indicated by the arrow in the MDI image of the top row of Fig. 2). Note that the absolute value of the patch's magnetic flux increases by a factor of 1.25 from 2000 July 13, 00:00 UT until 04:51 UT (when the last MDI image before the two events was obtained). The eastern components are located very close to a sunspot's gyroresonance source and show degrees of circular polarization of about $-58 \%$ and $-65 \%$ in the 05:25 and 05:40 UT events, respectively. The two radio sources are connected with somewhat diffuse loop-like soft $\mathrm{X}$-ray emission.

In the 05:25 UT event the western radio source is associated with a compact unresolved source in hard X-ray images made from data from the Hard X-ray Telescope (HXT) on board Yohkoh satellite (no HXT images can be made for the 05:40 UT event). It is interesting that in both events the eastern source is only prominent at radio wavelengths. Only the western source is seen to flare in soft and hard X-rays. Indeed the intensity of the soft X-ray emission which bridges the two radio components does not change significantly from 05:24:55 UT until the end of the 05:40 UT event. On the other hand the intensity time profile of the compact SXT brightening associated with the western sources follows quite well the variability of the soft X-ray flux recorded by GOES. The time profiles (see Figs. 3 and 4) show that in both events the short-duration burst components of the radio light curves of the two sources match well indicating clearly that the sources are magnetically connected.

\subsection{Event of 2000 July 13, 06:11 UT}

The radio event is associated with a relatively small loop which is resolved in the SXT images. The event occurred in AR9070, and we present it in Fig. 5. The soft X-ray loop shows brighter emission at its footpoints. This morphology and the loop shape does not change until the end of the flare. The soft X-ray plasma parameters show that at 06:11:25 UT the average temperature and electron density over the loop are about $12 \mathrm{MK}$ and $8 \times$ $10^{10} \mathrm{~cm}^{-3}$, respectively. 


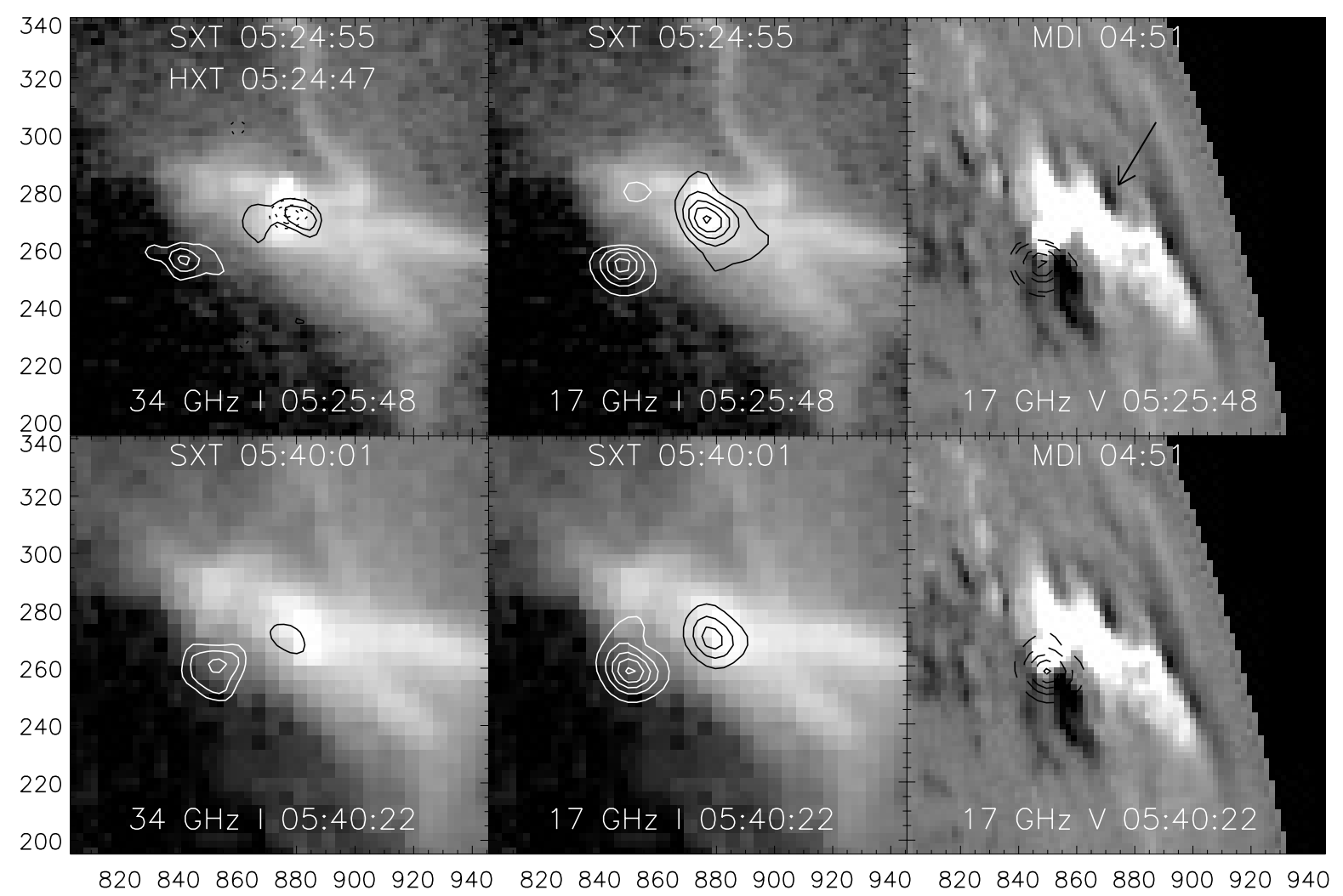

Fig. 2. Top row: the 2000 July 13, 05:25 UT event. From left to right: $34 \mathrm{GHz} I, 17 \mathrm{GHz} I$ and $17 \mathrm{GHz} V$ images at the peak of the radio burst overlaid on an SXT image and MDI magnetogram. Contours are plotted at 15\%, 35\%, 55\%, 75\%, and 95\% of the respective peaks in the $17 \mathrm{GHz} I\left(4 \times 10^{5} \mathrm{~K}\right)$ and $17 \mathrm{GHz} V\left(-2.1 \times 10^{5} \mathrm{~K}\right)$ images while the $34 \mathrm{GHz} I$ contours are plotted at $35 \%, 75 \%$, and $95 \%$ of the maximum $\left(10^{5} \mathrm{~K}\right)$. In the left image the dotted contours show a 14-23 keV channel HXT image (contour levels at 20\%, 50\% and $80 \%$ of the maximum hard X-ray emission). Bottom row: the 2000 July 13, 05:40 UT event. The format is identical to the top row. The maxima of the $17 \mathrm{GHz} I$, $V$ and $34 \mathrm{GHz} I$ maps are $7 \times 10^{5} \mathrm{~K},-4 \times 10^{5} \mathrm{~K}$, and $2 \times 10^{5} \mathrm{~K}$, respectively.

The radio emission covers the whole loop at $17 \mathrm{GHz}$ while it is somewhat less extended at $34 \mathrm{GHz}$. The $17 \mathrm{GHz} I$ emission peak lies over the southern footpoint of the soft X-ray loop while the $17 \mathrm{GHz} V$ and the $34 \mathrm{GHz} I$ peaks are almost coincident and displaced about 5 " northwards with respect to the $17 \mathrm{GHz} I$ peak. The degree of circular polarization at the peak of the radio event is between -20 and $-30 \%$. The 17 and $34 \mathrm{GHz} I$ time profiles show good agreement, which suggests that the same population of energetic electrons is responsible for both the 17 and $34 \mathrm{GHz}$ emissions.

\section{Model computations}

The time profiles of the radio bursts presented in Sect. 3 suggest that the 17 and $34 \mathrm{GHz}$ emission is gyrosynchrotron from nonthermal electrons spiraling along coronal magnetic field lines. A detailed quantitative study of the physical parameters controlling the radio burst emission requires comparison of the observations with models of gyrosynchrotron loop emission. Such comparison can be done only for the 2000 July 13, 06:11 UT event which shows a resolved flaring loop structure in soft X-rays. The 1999 July 30 event comes from an unresolved loop whose shape cannot be determined. On the other hand the 2000 July 13 05:25 and 05:40 UT events involve more than one loop (see Sect. 5.1, below) and comparison with single loop gyrosynchrotron emission models is not straightforward.

Here we use a gyrosynchrotron model loop in which the magnetic field has the form of a line dipole. The field lines are circles with a common tangent point at the dipole and the magnetic field falls off quadratically with distance from the line dipole. The loop is filled with energetic electrons with a power-law energy distribution and isotropic pitch angle distribution. The gyrosynchrotron emission from the loop is computed using a code in which the gyrosynchrotron emissivity and opacity are calculated exactly at specific points along the loop and the emitted radio emission flux is computed using simple radiative transfer. The model has been presented in detail by Nindos et al. (2000) and Kundu et al. (2001b) and the interested reader may refer to these articles for a detailed description of how the model's input parameters affect the resulting gyrosynchrotron emission (similar models have been developed by Preka-Papadema \& Alissandrakis 1992 and Bastian et al. 1998). The best-fit model should satisfy two criteria: (1) the projection of the model loop shape on the plane of the sky should be consistent with the appearance of the flaring loop in soft X-rays; and (2) the resulting model radio emission should reproduce both the observed fluxes and structures of the 17 and $34 \mathrm{GHz}$ emissions at the peak of the event. The comparison between the spatial structure of each model and the 


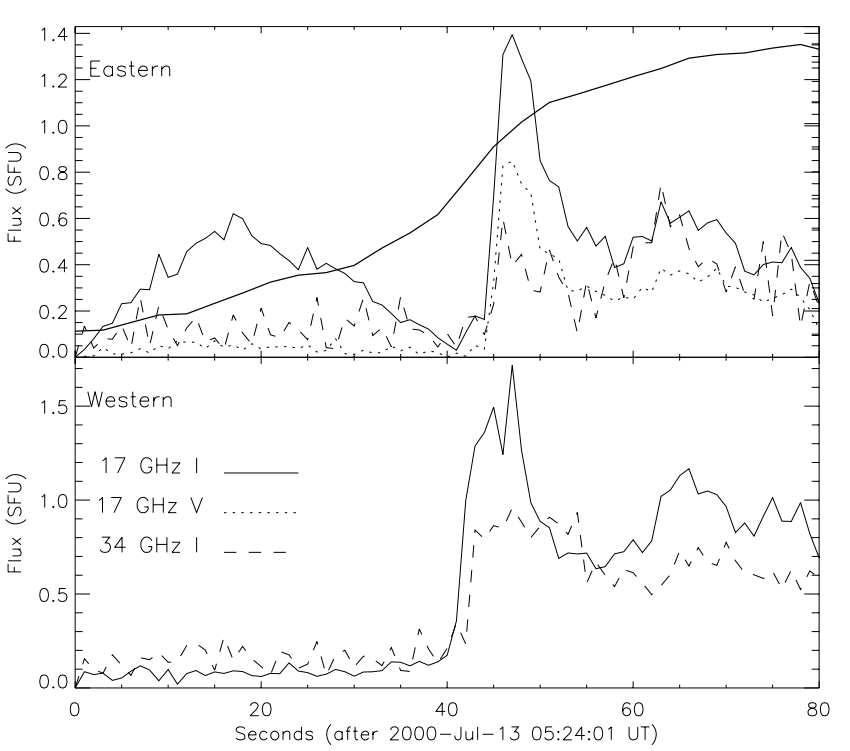

Fig. 3. Time profiles of the 05:25 UT event flux at $17 \mathrm{GHz} I$ and $V$ (absolute value of $V$ flux) and $34 \mathrm{GHz} I$. The top panel shows the eastern radio source and the bottom panel shows the western radio source. In the top panel we present also the flare's total soft X-ray flux measured by the GOES satellite in the $1.5-10 \mathrm{keV}$ channel.

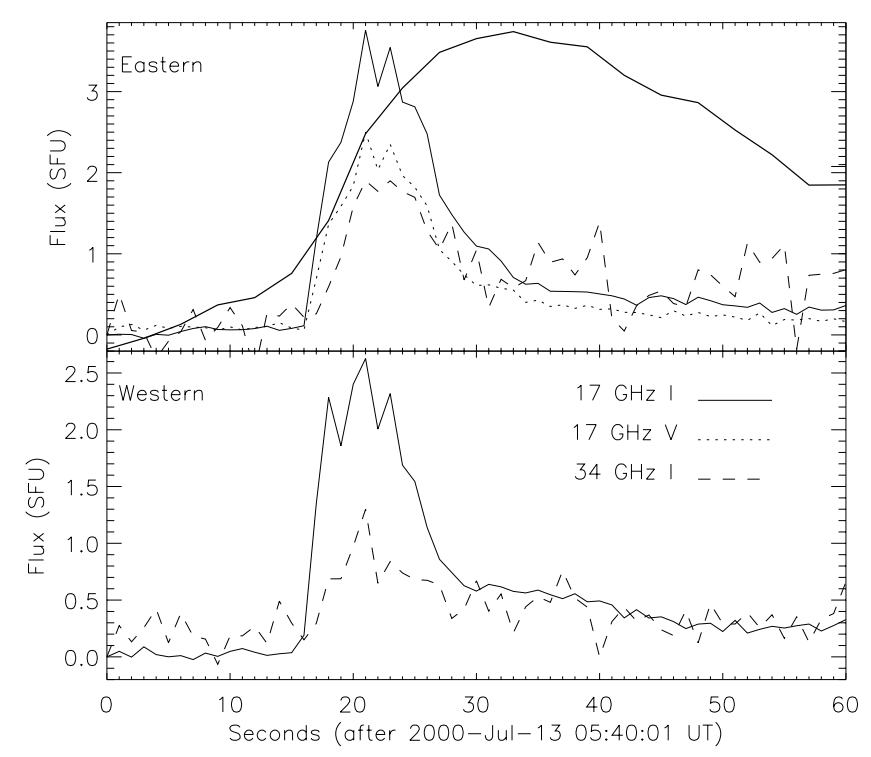

Fig. 4. Same as in Fig. 3 for the 05:40 UT event.

observations has been done after we have convolved the model emission with the appropriate NoRH beam.

The model loop is located at the heliographic coordinates of the observed flaring loop. First we determine the loop geometry parameters (azimuth, tilt, footpoint separation and maximum height of the model loop), that match best the observed loop shape. These parameters are presented in Table 2. Data from the Nobeyama Polarimeter indicate that the turnover frequency for this event lies between 3.75 and $9.4 \mathrm{GHz}$. Consequently both the 17 and $34 \mathrm{GHz}$ emissions must be optically thin. Using the 17 and $34 \mathrm{GHz}$ maximum fluxes we compute the slope $\alpha$ of the optically thin part of the spectrum. Since the emission is optically thin, $\alpha$ yields directly the energy spectral index $\delta$ of the
Table 2. Best Fit Model Parameters for the 2000 July 13, 06:11 UT Flare.

\begin{tabular}{lr}
\hline \hline Parameter & Value \\
\hline Loop orientation with respect to local North & $40^{\circ}$ \\
Angle between the loop plane and local vertical & $0^{\circ}$ \\
Footpoint separation & $16^{\prime \prime}$ \\
Maximum height of loop & $7.7^{\prime \prime}$ \\
Loop thickness at maximum height & $6.3^{\prime \prime}$ \\
Loop transverse dimension & $5.3^{\prime \prime}$ \\
Footpoint and looptop magnetic field strength & $800 / 665 \mathrm{G}$ \\
Energy spectral index of energetic electrons & 2.4 \\
Density of energetic electrons & $5.5 \times 10^{4} \mathrm{~cm}^{-3}$ \\
Low and high-energy cutoffs of energetic electrons & $10 / 10000 \mathrm{keV}$ \\
\hline
\end{tabular}

radio-emitting electrons ( $\alpha=1.22-0.90 \delta$; Dulk 1985). We find that $\delta=2.4$. Then the best-fit model to the radio observations is obtained when the remaining input parameters (density and low- and high-energy cutoffs of the radio-emitting electrons, photospheric footpoint magnetic field strength, loop thickness at the location of the loop's maximum height, and transverse dimension of the loop) have the values appearing in Table 2. Note that the photospheric magnetic field strength is a free parameter because the flare occurred close to the limb and only line-of-sight magnetograms are available.

The resulting best-fit model $I$ total fluxes are 46 and $22 \mathrm{sfu}$ at 17 and $34 \mathrm{GHz}$, respectively, in excellent agreement with the observations. The absolute value of the $17 \mathrm{GHz} V$ total flux is $19 \mathrm{sfu}$, a factor of 1.4-1.9 higher than the observed. Figure 6 shows the structure of the best-fit model emission at 17 and $34 \mathrm{GHz}$ before convolution with the appropriate NoRH beams. Both 17 and $34 \mathrm{GHz} I$ maps show peaks close to the footpoints of the loop. This is consistent with the typical appearance of the optically thin emission at high frequencies. However, these peaks are assymetric; the southern being stronger. Note also that some emission (of the order of $10-40 \%$ of the maximum $I$ peaks) between the footpoints exists at both frequencies. The $V$ model maps peak close to the northern footpoint because the angle between the magnetic field and the line of sight is smaller there. However, the overall distribution of $V$ along the model loop is somewhat more uniform than the distribution of $I$. This reflects the fact that the difference between the extraordinarymode and the ordinary-mode emission does not change dramatically along the loop.

When we convolve the structures appearing in Fig. 6 with the NoRH beams we obtain the maps of Fig. 7. Their morphological resemblance with the NoRH maps of Fig. 5 is very good. The convolution washes out the northern emission peaks resulting in $I$ maps with peaks close to the southern footpoint of the loop. We are also able to reproduce the displacement of the peak of the $17 \mathrm{GHz} V$ map with respect to the $17 \mathrm{GHz} I$ peak, but not the similar displacement of the $34 \mathrm{GHz} I$ peak. Also in the models of Fig. 7 the degree of circular polarization at $17 \mathrm{GHz}$ is between $25-50 \%$ with an average of about $40 \%$, i.e. higher than the observations. The overall good agreement betwen the morphology of the best-fit models and the 


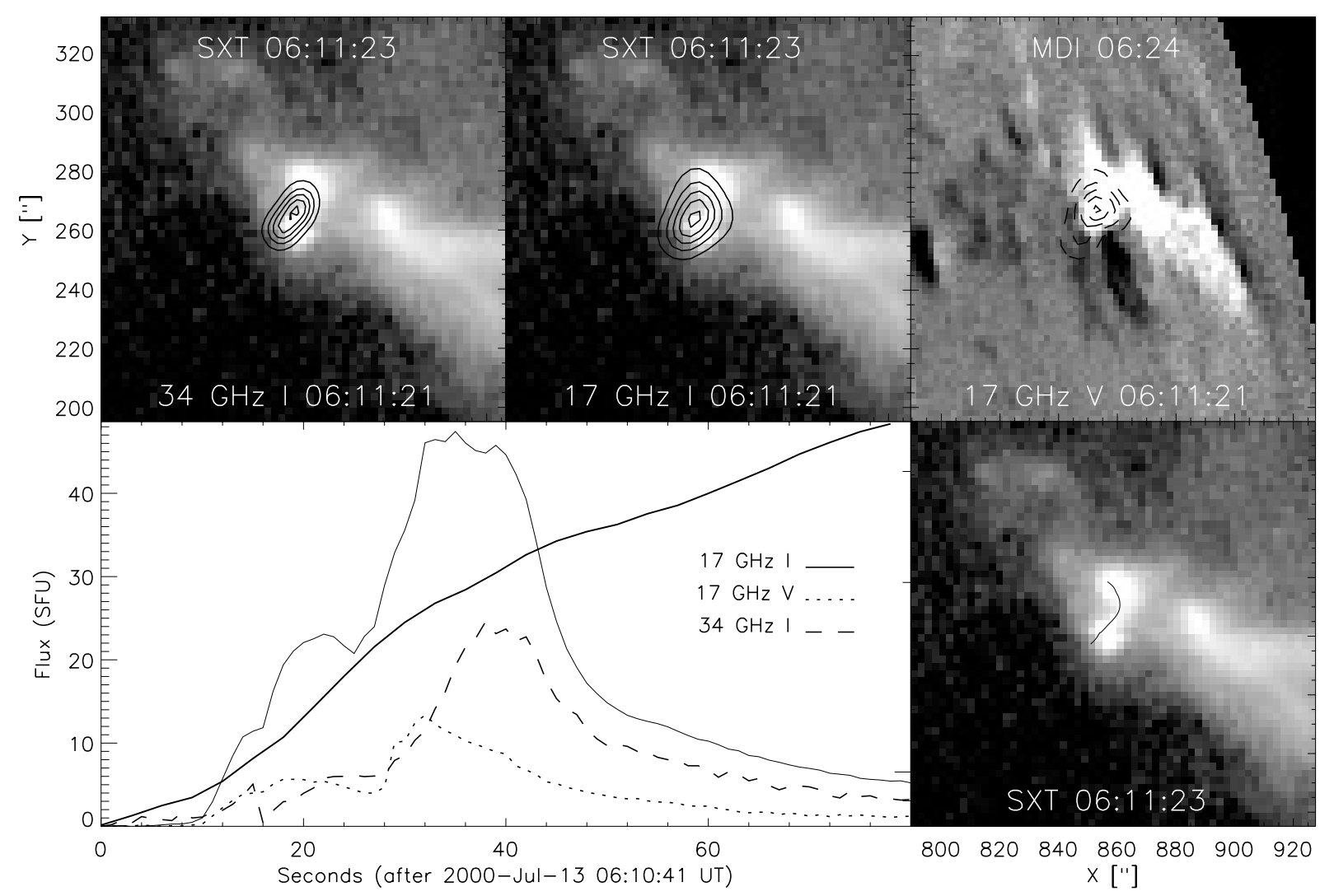

Fig. 5. The 2000 July 13, 06:11 UT event. Top row from left to right: $34 \mathrm{GHz} I$ and $17 \mathrm{GHz} I$ and $V$ images at the peak of the event overlaid on an SXT image and an MDI magnetogram. Bottom left: time profiles of the event's radio fluxes and of the soft X-ray flux measured by GOES. Note that we give the absolute value of the $V$ flux. Bottom right: the SXT image presented in the top row with a solid curve which outlines the flaring loop shape. Contours are plotted at $15 \%, 35 \%, 55 \%, 75 \%$, and $95 \%$ of the respective peaks in the $17 \mathrm{GHz} I\left(7.6 \times 10^{6} \mathrm{~K}\right), 17 \mathrm{GHz} V$ $\left(-2.1 \times 10^{6} \mathrm{~K}\right)$ and $34 \mathrm{GHz} I\left(2.5 \times 10^{6} \mathrm{~K}\right)$ images.

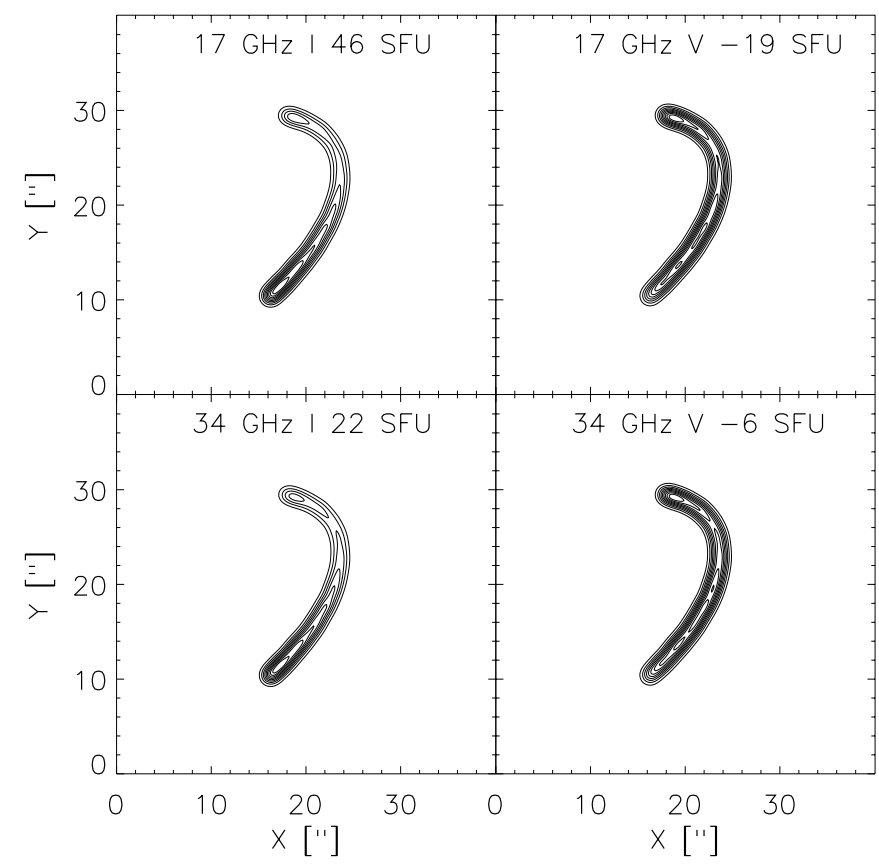

Fig. 6. 17 and $34 \mathrm{GHz} I$ and $V$ maps resulting from our best-fit gyrosynchrotron model to the radio data. The maps are presented before their convolution with the appropriate NoRH beam sizes. Contours are plotted at $10 \%, 20 \%, \ldots, 90 \%$ of each map peak. observations is demonstrated in the two bottom panels of Fig. 7 where we plot the radio emission profiles along the model loop and the emission profiles of the NoRH maps presented in Fig. 5 along the solid black curve shown in the bottom right panel of Fig. 5.

\section{Discussion}

\subsection{The 2000 July $13,05: 25$ and 05:40 UT flares}

The flare configuration of the 05:25 and 05:40 UT events is clearly different from the single compact bipolar loop configuration that appears in the 1999 July 30 event and proposed by Kundu et al. (2001c) for the five spiky events they studied. Here we shall discuss possible alternatives for the configuration of these two events. Our data rule out the possibility that each event takes place in a single long loop connecting the two radio sources because: (1) the time variability of the diffuse loop-like soft X-ray emission connecting the two radio sources is below the $3 \sigma$ level of the uncertainties due to photon statistics; and (2) at the same time the variability of the soft X-ray intensity from the compact bright loop associated with the western sources follows well the time profile behavior of the soft X-ray flux derived from the GOES data. Since in both events the radio components are located above mixed polarity photospheric fields, 

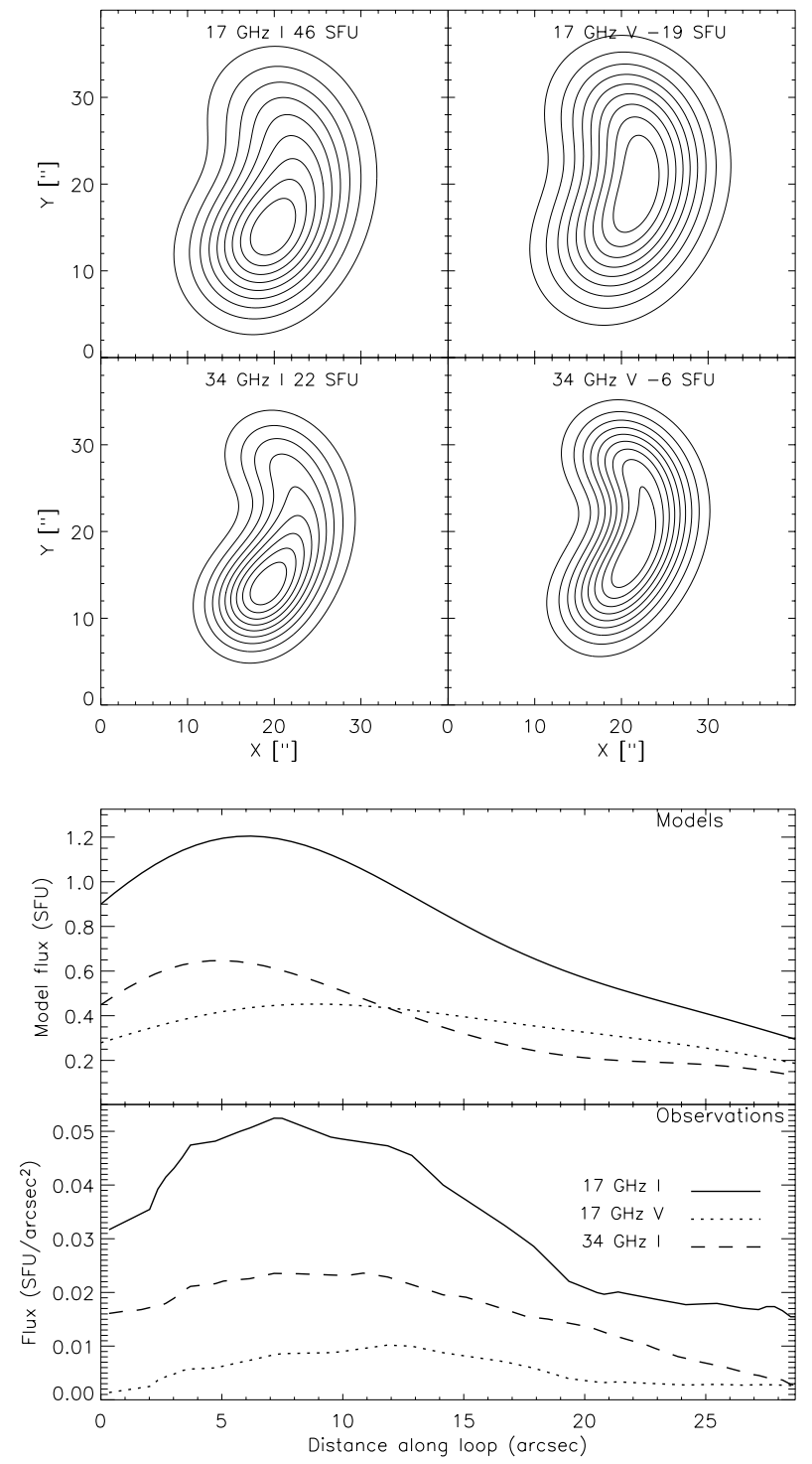

Fig. 7. First and second row: the maps of Fig. 6 after their convolution with the appropriate NoRH beams. Contours are plotted at $10 \%$, $20 \%, \ldots, 90 \%$ of each map peak. Third row: spatial profiles of the radio emissions from the best-fit model as a function of distance along the model loop. Bottom row: one-dimensional representation of the 06:11 UT event at the time of maximum. These profiles have been computed along the solid black curve shown in the right bottom frame of Fig. 5. For a better comparison, we have divided the NoRH values with the appropriate beam.

one might speculate that each component occurs in a compact bipolar flaring loop. However, this scenario is not acceptable because (1) there is no EUV, or soft X-ray or hard X-ray flaring emission associated with the eastern sources and (2) our calculations show that the distribution of the degree of circular polarization over the eastern sources is flat. This is also evident from a direct visual inspection of Fig. 2: in each event the shape of the $17 \mathrm{GHz} I$ eastern source is similar to the shape of the $17 \mathrm{GHz} V$ source and there is no displacement between the location of their peaks. However, an unresolved bipolar source even in the case where a unique sense of polarization is observed along the compact flaring loop should show a slope in the spatial distribution of its degree of circular polarization.

The most probable interpretation for the 05:25 and 05:40 UT events is that two sets of loops are involved in the flare production. Both events share some common features with the "double-loop configuration" flares described by Hanaoka (1996, 1997): (1) they involve a small relatively compact loop which flares up in soft X-rays; (2) a larger fainter loop-like soft $\mathrm{X}$-ray structure connects the two radio components; (3) flux emergence of parasitic polarity is associated with the radio component which is related to the small bright loop; (4) only one of the radio sources would be identified as a flare site at other wavelengths (i.e. the source associated with the bright small loop) while the western radio sources are not prominent at any other wavelength range.

Furthermore, morphological discrepancies between some properties of our events with the typical morphology described by Hanaoka do not necessarily contradict the above interpretation: (1) the typical photospheric magnetic field configuration in the Hanaoka's events shows a "bipolar and remote unipolar" structure, namely the radio component associated with the small bright loop lies over a bipolar region while the other component lies over a unipolar region. Clearly this does not happen here. We note however, that while flux emergence of parasitic polarity occurs at the location of the western sources associated with the bright compact loop, no flux emergence occurs below the eastern components; (2) the diffuse loop-like soft $\mathrm{X}$-ray emission which bridges the two radio components does not show any significant brightening at the decay phase of the events. But this is also the case for about half of the events analyzed by Hanaoka (1997); (3) Hanaoka (1997) inferred that the radio component associated with the small bright loop actually includes two oppositely polarized microwave sources because the distribution of its degree of polarization has a slope which cannot be produced by a single source. In our two events the western sources are practically unpolarized. The two flares occur close to the limb and the direction of the magnetic field with respect to the line of sight may be large enough resulting in very low circular polarization. The increase of the absolute value of the magnetic flux of the negative polarity patch (indicated by the arrow in Fig. 2) implies that the interaction of loops with different sizes may be responsible for the two events.

\subsection{The 2000 July $13,06: 11$ UT flare}

The resolved soft X-ray flaring loop allowed us to check the properties of the flare's radio emission against gyrosynchrotron loop models. The parameters of the model loop geometry were constrained by the shape of the soft X-ray loop on the plane of the sky. The radio observations provided the energy spectral index of the radio-emitting electrons. The other model input parameters were unconstrained. The best-fit model parameters appear in Table 2 and overall reproduce satisfactorily the fluxes and spatial structures of the 17 and $34 \mathrm{GHz}$ emission at the event maximum.

The variation of the magnetic field along the model loop is relatively small: $800 \mathrm{G}$ at the footpoints and $665 \mathrm{G}$ at the loop 
top. This feature of the model is essential for the reproduction of the radio spatial structures. A larger variation of the magnetic field along the loop can be achieved if we either increase the loop height or decrease its footpoint separation. But then we come up with a loop shape that is not consistent with the SXT loop and the resulting radio emission after convolution with the appropriate NoRH beams shows two distinct peaks above the footpoints at both frequencies. We also note that both the loop thickness at the location of its maximum height and the loop transverse dimension are factors of 0.4 and 0.3 , respectively, of the footpoint separation. Our previous radio burst modeling attempts (Nindos et al. 2000) suffered from the problem that these factors were much smaller $(0.04$ and 0.02 , respectively) suggesting that the model loop was very thin. In the present study we do not have to confront such difficulties. Takahashi (1997) has found that the aspect ratio of flaring loops is usually $0.1-1$ and therefore our model loop is consistent with his results. Our model requires the presence of highenergy electrons (up to $10 \mathrm{MeV}$ ). If we remove the electrons above about 500-700 keV the model fluxes decrease significantly, especially at the higher frequency (see White \& Kundu 2000). Our model yields an upper limit of about $1 \mathrm{erg} \mathrm{cm}^{-3}$ for the energy of the nonthermal radio-emitting electrons at the peak of the radio event. However, we point out that the energy of the precipitating nonthermal electrons producing hard $\mathrm{X}$-rays is not included in this rough estimate. At the same time the plasma parameters derived from the SXT data flare yield a rough estimate of thermal energy of about $200 \mathrm{erg} \mathrm{cm}^{-3}$ in the soft $\mathrm{X}$-ray source. The plasma $\beta$ in the flaring model loop is $5.2-7.6 \times 10^{-3}$.

The basic problem of our best-fit model is that it yields somewhat higher degrees of circular polarization than the observations. These discrepancies disappear if we change some of the model input parameters appropriately but then either the loop shape is not consistent with the soft X-ray loop or the transverse loop dimension should become implausibly large compared to the loop thickness in order to reproduce the observed fluxes. We believe that the polarization discrepancies may come from inhomogeneities that have not been taken into account in our simplified loop geometry.

Our model is merely the best-fit model at the peak of the radio event. We cannot follow the temporal evolution of the flare because we do not have suitable data to determine the energetic electron injection rate. We note however that since the density of the loop's thermal plasma is high, collisions may play an important role in the time evolution of the radio emission. Following Spitzer (1964) we find that from the peak of the event until the radio emission decreases to one-half of its peak value collisions thermalize electrons up to about $500 \mathrm{keV}$.

\section{Summary and conclusions}

Our study of four short-duration simple microwave bursts for which partial SXT coverage was available revealed important properties of the flare configurations. In a previous study of similar short-duration microwave events, Kundu et al. (2001c) inferred from the NoRH maps and MDI magnetograms that these bursts are consistent with a single compact loop configuration. One of the events we studied, indeed came from a compact loop which was unresolved in the SXT images.

Another event originated from a single loop that was resolved in soft X-rays. This allowed us to study in detail the physical properties of the flaring loop by comparing the radio data at the peak of the event with gyrosynchrotron loop models. The SXT images constrained the loop geometry input parameters because the projection of the model loop on the plane of the sky should resemble the soft X-ray loop. Data from the Nobeyama polarimeter, indicated that both 17 and $34 \mathrm{GHz}$ emissions were optically thin, consequently the 17 and $34 \mathrm{GHz}$ fluxes gave information about the energy spectral index of the radio-emitting electrons. With our best-fit model we were able to derive a self-consistent picture of the flare site because we reproduced satisfactorily both the observed loop shape as well as most of the features of the radio emission. The flaring loop was relatively small with footpoint separation of $16^{\prime \prime}$ and maximum height of 7.7". The loop thickness at the position of maximum height was $6.3^{\prime \prime}$ while its transverse dimension was 5.3". The loop was filled with energetic electrons with energy spectral index of 2.4 , density of $5.5 \times 10^{4} \mathrm{~cm}^{-3}$ and low and high-energy cutoffs at $10 \mathrm{keV}$ and $10 \mathrm{MeV}$, respectively. Two important features of the flaring loop were that (1) the variation of the magnetic field between the footpoints and the looptop was small; and (2) electrons with energies up to $10 \mathrm{MeV}$ were present.

The remaining two events (05:25 and 05:40 UT events) were not consistent with a single-loop configuration. At both frequencies there were two sources with similar time profiles. One of them was cospatial with a bright compact soft X-ray loop which was associated with a patch of parasitic magnetic polarity whose photospheric magnetic field flux increased before the two flares. The other source was prominent only at 17 and $34 \mathrm{GHz}$; no flaring associated with it could be identified in EUV or soft/hard X-ray data. The two sources were bridged by diffuse loop-like soft X-ray emission whose intensity did not change significantly during the observations. From these features we infer that these events are similar to Hanaoka's (1996, 1997) "double-loop configuration" flares, i.e. the interaction of two sets of loops with different sizes may be responsible for the flares (see also Kundu et al. 2001a). Therefore the simple short-duration microwave bursts do not always appear in the same configuration. Some of them appear as single-loop events but it is possible that some events are caused by two interacting loops.

Acknowledgements. This research at the University of Maryland was carried out with support from NSF grants ATM 02-33907 and NASA grant NAG 5-12860. Part of this research was done while AN visited the University of Maryland. He takes the opportunity to express his thanks to the solar group for their warm hospitality. The work in Irkutsk was partly supported by Russian RFBR grants 03-02-16591 and 477.2003.2. We thank Dr. S. M. White for useful discussions and for his comments on the manuscript.

\section{References}

Alissandrakis, C. E. 1986, Sol. Phys., 104, 207

Alissandrakis, C. E., Nindos, A., \& Kundu, M. R. 1993, Sol. Phys., 147,343 
Bastian, T. S., \& Kiplinger, A. 1991 in Max 1991 Workshop 3, ed. R. Winglee, \& A. Kiplinger (Boulder: Univ. Colorado), 153

Bastian, T. S., Benz, A. O., \& Gary, D. E. 1998, ARA\&A, 36, 131

Dulk, G. A. 1985, ARA\&A, 23, 169

Hanaoka, Y. 1996, Sol. Phys., 165, 275

Hanaoka, Y. 1997, Sol. Phys., 173, 319

Holman, G. D., Kundu, M. R., \& Papadopoulos, K. 1982, ApJ, 257, 354

Kundu, M. R., Schmahl, E. J., Velusamy, T., \& Vlahos, L. 1982, A\&A, 108, 188

Kundu, M. R., Grechnev, V. V., Garaimov, V. I., \& White, S. M. 2001a, ApJ, 563, 389

Kundu, M. R., Nindos, A., White, S. M., \& Grechnev, V. V, 2001b, ApJ, 557, 880

Kundu, M. R., White, S. M., Shibasaki, K., Sakurai., T., \& Grechnev, V. V. 2001c, ApJ, 547, 1090

Nakajima, H., Nishio, M., Enome, S., et al. 1994, Proc. IEEE, 82, 705
Nindos, A., White, S. M., Kundu, M. R., \& Gary, D. E. 2000, ApJ, 533, 1053

Nishio, M., Yaji, K., Kosugi, T., Nakajima, H., \& Sakurai, T. 1997, ApJ, 489, 976

Preka-Papadema, P., \& Alissandrakis, C. E. 1992, A\&A, 257, 307

Shevgaonkar, R. K., \& Kundu, M. R. 1985, ApJ, 292, 733

Spitzer, L. Jr. 1964, Physics of Fully Ionized Gas (New York: Wiley)

Takahashi, M. 1997, Ph.D. Thesis, Graduate Univ. Adv. Study (NAOJ), Tokyo

White, S. M. 1994, in High Energy Solar Phenomena: A New Era of Spacecraft Measurements, ed. J. Ryan, \& W. T. Vestrand (New York: AIP), AIP Conf. Proc., 294, 199

White, S. M., \& Kundu, M. R. 2000, in High Energy Solar Physics: Anticipating HESSI, ed. R. Ramaty, \& N. Mandzhavidze (San Francisco: ASP), ASP Conf. Ser., 206, 335

White, S. M., Kundu, M. R., Garaimov, V. I., Yokoyama, T., \& Sato, J. 2002, ApJ, 576, 505 